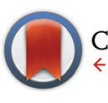

CrossMark \& click for updates

Cite this: Polym. Chem., 2015, 6 , 7112

Received 23rd July 2015

Accepted 20th August 2015

DOI: 10.1039/c5py01162k

www.rsc.org/polymers

\title{
Water-soluble and redox-responsive hyperbranched polyether copolymers based on ferrocenyl glycidyl ether $\uparrow$
}

\author{
Arda Alkan, $t^{a}$ Rebecca Klein, $t^{\text {b,c }}$ Sergii I. Shylin, ${ }^{d}$ Ulrike Kemmer-Jonas, ${ }^{b}$ \\ Holger Frey ${ }^{\mathrm{b}}$ and Frederik R. Wurm*a
}

\begin{abstract}
Water-soluble copolymers of ferrocenyl glycidyl ether ( $f c G E$ ) and glycidol were prepared via anionic ringopening multibranching polymerization (ROMBP). The resulting hyperbranched materials with molecular weights $\left(M_{n}\right)$ of 3500 to $12300 \mathrm{~g} \mathrm{~mol}^{-1}$ and relatively narrow molecular weight distributions $\left(M_{w} / M_{n}=\right.$ 1.40-1.69) exhibit both temperature- as well as redox-responsive behavior, which was studied via turbidity measurements. The cloud point temperatures $\left(T_{c}\right)$ were adjusted between 45 and $60{ }^{\circ} \mathrm{C}$ through variation of the fCGE comonomer content. Additionally, these $T_{C} S$ can be increased by the addition of an oxidizing agent. The extent of oxidation of the materials was quantified by Mößbauer spectroscopy and can be correlated to the change in the $T_{\mathrm{C}} \mathrm{s}$. Furthermore, the copolymers were investigated via ${ }^{1} \mathrm{H}$ and inverse gated (IG) ${ }^{13} \mathrm{C}$ NMR spectroscopy, size exclusion chromatography (SEC), MALDI-ToF mass spectroscopy and differential scanning calorimetry (DSC). The reversible oxidation of the fc moities is demonstrated by cyclic voltammetry.
\end{abstract}

\section{Introduction}

Organometallic polymers combine the unique properties of inorganic materials, such as redox activity with polymer properties. In such polymers, metals are often incorporated in stable 18 electron sandwich-complexes to ensure durable polymers in bulk and in solution. Metallocenes, such as ferrocene (fc), can be incorporated either in the polymer backbone or as side chains, the most prominent examples being poly(ferrocenylsilane) (PFS) $)^{1,2}$ and poly(vinylferrocene) (PVfc). ${ }^{3}$ Also organometallic acrylates have been reported. ${ }^{4}$ Recently, our group introduced the first fc-containing epoxide monomer for oxyanionic ring-opening polymerization, i.e. ferrocenyl methyl glycidyl ether (fcGE). ${ }^{5-7}$ This epoxide monomer was

\footnotetext{
${ }^{a}$ Max Planck Institute for Polymer Research (MPIP), Ackermannweg 10, 55128 Mainz, Germany.E-mail:wurm@mpip-mainz.mpg.de; Fax: +49 6131370 330; Tel: +496131379581

${ }^{b}$ Institute of Organic Chemistry, Johannes Gutenberg University, Duesbergweg 10-14, 55128 Mainz, Germany

${ }^{c}$ Graduate School "Materials Science in Mainz", Staudingerweg 9, 55128 Mainz, Germany

${ }^{d}$ Institute of Inorganic and Analytical Chemistry, Johannes Gutenberg University, Duesbergweg 10-14, 55128 Mainz, Germany

$\dagger$ Electronic supplementary information (ESI) available: Fig. S1-S11, NMR, SEC, MALDI-ToF MS, Mößbauer spectroscopy, cyclic voltammetry. See DOI: 10.1039/ c5py01162k

$\$$ These authors contributed equally.
}

copolymerized with ethylene oxide (EO) to generate watersoluble, multi-stimuli responsive materials.

While there are many studies on linear ferrocene-containing polymers, branched materials with fc-units have been reported only rarely, ${ }^{8}$ some works have focused on fc-dendrimers. ${ }^{9}$ In recent years hyperbranched (hb) polymers have found increasing attention as randomly branched analogues of dendrimers. To the best of our knowledge only very few, albeit hydrophobic ferrocene-containing hyperbranched polymers, based on hyperbranched poly(carbosilane)s (hbPCSs), ${ }^{10,11}$ poly(3-ethyl-3-(hydroxymethyl)-oxetane)s (hbPOs) ${ }^{12}$ and poly(phenylene)s (hbPPs), ${ }^{13}$ have been reported to date.

Hyperbranched polymers offer potential, e.g., for biomedicine, catalysis, membrane materials and coatings. ${ }^{14-16}$ One benefit in comparison to the perfectly branched dendrimers is their accessibility in facile one-step syntheses even on larger scales, opening their use for industrial applications. ${ }^{17}$ Hyperbranched polyglycerol (hbPG), which is synthesized via anionic ring-opening multibranching polymerization (ROMBP) of glycidol, a latent cyclic $\mathrm{AB}_{2}$ monomer, is a highly interesting material due to its water-solubility, the flexible polyether backbone, ${ }^{18,19}$ high functionality and excellent biocompatibility. $^{20,21}$ The synthesis of hbPG via slow monomer addition (SMA) enables control over molecular weights (6000 to $24000 \mathrm{~g}$ $\left.\mathrm{mol}^{-1}\right)$, relatively narrow molecular weight distributions $(\nexists=$ 1.3-1.8) and high degree of branching. ${ }^{22,23}$ Copolymerization of glycidol with (functional) glycidyl ethers ${ }^{24}$ or the post- 


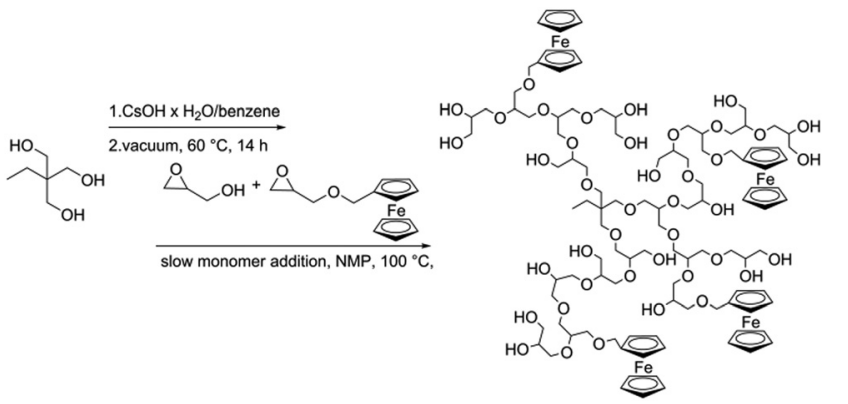

Scheme 1 Synthetic $A B / A B_{2}$ copolymerization strategy for watersoluble hbP[G-co-fcGE] copolymers.

polymerization modification of hbPG allows for the synthesis of stimuli-responsive hbPG showing temperature-, ${ }^{25-28}$ $\mathrm{pH}-{ }^{29-31}$ or redox-responsive behavior. ${ }^{32}$

In this work, the copolymerization of glycidol and fcGE is introduced (Scheme 1), enabling the one-pot synthesis of multi-stimuli-responsive materials. The combination of the hbPG backbone with multiple hydroxyl groups provides excellent water-solubility and, in combination with the hydrophobic fc groups, temperature-dependent solubility of the copolymers in aqueous solution is achieved, even higher than for the already reported linear fc-containing poly(ethylene glycol)s $\mathrm{P}[\mathrm{fcGE}-\mathrm{co}-\mathrm{EO}] .{ }^{5}$ Furthermore, the iron centers in the fc units are redox-responsive and can be oxidized reversibly from Fe(II) to $\mathrm{Fe}(\mathrm{III})$ enabling to vary the hydrophilicity of the copolymers.

\section{Experimental}

\section{Reagents}

All solvents and reagents were purchased from Acros Organics or Sigma Aldrich and used as received, unless otherwise stated. Glycidol and $N$-methyl-2-pyrrolidone (NMP) were freshly distilled from $\mathrm{CaH}_{2}$ prior to use. fcGE was synthesized according to the published procedures, purified via column chromatography and dried by azeotropic distillation of benzene to remove traces of water. ${ }^{5}$

\section{Instrumentation}

${ }^{1} \mathrm{H}$ and ${ }^{13} \mathrm{C}$ NMR spectra were recorded at 400 and $100 \mathrm{MHz}$ on a Bruker Avance II 400 NMR spectrometer. For SEC measurements in DMF (containing $0.25 \mathrm{~g} \mathrm{~L}^{-1}$ of LiBr), an Agilent 1100 series was used as an integrated instrument including a PSS Gral column $\left(10^{6} / 10^{4} / 10^{2} \AA\right.$ porosity $)$ and a RI detector. Calibration was achieved using poly(ethylene glycol) (PEG) standards provided by PSS. DSC measurements were performed using a PerkinElmer 8500 thermal analysis system and a PerkinElmer CLN2 thermal analysis controller in the temperature range from -90 to $+100{ }^{\circ} \mathrm{C}$ with heating rates of $10 \mathrm{~K} \mathrm{~min}^{-1}$. MALDI-ToF MS measurements were performed using a Shimadzu Axima CFR MALDI-TOF mass spectrometer, employing dithranol (1,8,9-trishydroxy-anthracene) as a matrix.

\section{Turbidity measurements}

Cloud points were determined in deionized water $\left(5 \mathrm{~g} \mathrm{~L}^{-1}\right)$ and observed by optical transmittance of a light beam $(\lambda=500 \mathrm{~nm})$ through a $1 \mathrm{~cm}$ sample quartz cell. The measurements were performed in a Jasco V-630 photospectrometer with a Jasco ETC-717 Peltier element. The intensity of the transmitted light was recorded versus the temperature of the sample cell. The heating/cooling rate was $5 \mathrm{~K} \mathrm{~min}^{-1}$, and values were recorded in $1 \mathrm{~K}$ steps.

\section{Cyclic voltammetry (CV)}

CV measurements of the copolymer samples were carried out in a conventional three-electrode cell using a WaveDriver 20 bipotentiostat (Pine Intrument Company, USA) and deionized water as solvent for polymer solutions with $5 \mathrm{~g} \mathrm{~L}^{-1}$ concentration. Potassium chloride at concentrations of $0.1 \mathrm{M}$ was used as supporting electrolyte. A glassy carbon disk served as working electrode. $\mathrm{Ag} / \mathrm{AgCl}$ and platinum wire were used as reference and counter electrodes, respectively.

\section{Mößbauer spectroscopy}

${ }^{57} \mathrm{Fe}-\mathrm{Mößbauer}$ spectra were recorded in transmission geometry with a ${ }^{57} \mathrm{Co}$ source embedded in a rhodium matrix using a conventional constant-acceleration Mößbauer spectrometer ("Wissel") equipped with a nitrogen gas-flow cryostat at $80 \mathrm{~K}$. The absorbers were prepared by freezing aqueous solutions of samples in plastic holders. Fits of the experimental data were performed using the Recoil software. ${ }^{33}$ Isomer shifts are given relatively to $\alpha$-Fe at ambient temperature.

\section{Synthesis of hyperbranched poly(glycerol-co-ferrocenyl glycidyl ether) (hbP[G-co-fcGE])}

In a dry Schlenk flask under argon atmosphere, 1,1,1-trimethylolpropane (TMP) as initiator was suspended with cesium hydroxide monohydrate ( 0.3 eq.) in $5 \mathrm{~mL}$ benzene and stirred for $30 \mathrm{~min}$ at $60^{\circ} \mathrm{C}$ under static vacuum. Then, remaining water impurities were removed by azeotrope distillation with benzene at $60{ }^{\circ} \mathrm{C}$ for $12 \mathrm{~h}$ under high vacuum. The initiator salt was dissolved in $0.5 \mathrm{~mL}$ NMP at $100{ }^{\circ} \mathrm{C}$. Glycidol and fcGE were dissolved in a separate flask in NMP to obtain a $50 \%$ dilution. The monomer solution was added slowly to the initiator solution over 6 hours via a syringe pump while vigorously stirring. After complete addition, it was stirred for another $30 \mathrm{~min}$ and methanol $(0.5 \mathrm{~mL})$ was added to terminate the polymerization. The solvents were removed under vacuum and the resulting polymer precipitated twice from methanol into cold diethyl ether, to obtain the product as brownish highly viscous liquid. Yields: $85-90 \% .{ }^{1} \mathrm{H}$ NMR (DMSO- $d_{6}$, $400 \mathrm{MHz}$ ): $\delta$ [ppm]: 4.80-4.40 (m, OH-groups), 4.23 (s, fc), 4.14 (s, fc), 3.76-3.19 (m, polyether backbone), $1.20\left(\mathrm{br}, 2 \mathrm{H}, \mathrm{CH}_{2^{-}}\right.$ TMP), 0.80 (br, 2H, $\mathrm{CH}_{3}$-TMP).

\section{Two-step synthesis of high molecular weight hyperbranched} poly(glycerol-co-ferrocenyl glycidyl ether) (hbP[G-co-fcGE])

A literature protocol using a macroinitiator was applied for the synthesis of high molecular weight polymers..$^{23}$ P1 $(100 \mathrm{mg}$, 
$\left.M_{\mathrm{n}}=3500 \mathrm{~g} \mathrm{~mol}{ }^{-1}, 6.1 \% \mathrm{fcGE}\right)$ and $\mathrm{CsOH} \cdot \mathrm{H}_{2} \mathrm{O}(19.1 \mathrm{mg}$, 0.1 eq.) were dissolved in benzene and dried for $15 \mathrm{~h}$ under high vacuum. The macroinitiator salt was dissolved in dry NMP (0.4 mL) at $100{ }^{\circ} \mathrm{C}$. Glycidol (338 mg) and fcGE (79 mg) were dissolved in a separate flask in NMP $(0.6 \mathrm{~mL})$. The monomer solution was added slowly to the initiator solution over 10 hours via a syringe pump while vigorously stirring. After complete addition, it was stirred for another $30 \mathrm{~min}$ and methanol $(0.5 \mathrm{~mL})$ was added to terminate the polymerization. The solvents were removed under vacuum and the resulting polymer precipitated twice from methanol into cold diethyl ether, to obtain the product as brownish highly viscous liquid. Yield: $60 \%$. NMR data match the above mentioned.

\section{Oxidation process of $\mathrm{hbP}[\mathrm{G}-\mathrm{co}$-fcGE]}

For partly oxidized P3, P3 (30 mg) was dissolved in dest. water and $0.5 \mathrm{eq}$. silver triflate $(11.5 \mathrm{mg})$ respective to ferrocene units were added. The solution was stirred for $30 \mathrm{~min}$ and filtered through a syringe filter to get rid of elementary silver. For the full oxidized P3 sample, an excess of silver triflate (1.05 eq.) was added to the polymer solution.

\section{Results and discussion}

\section{Synthesis and molecular weight determination}

In order to generate copolymers of glycidol and fcGE a synthetic procedure adopted from studies had to be devised. ${ }^{34}$ First, the trishydroxyfunctional initiator, trimethylol propane (TMP), was partly deprotonated (30 mol\%) with cesium hydroxide and - after the removal of reaction water - a mixture of glycidol and fcGE was added via slow monomer addition over 6 hours at $100{ }^{\circ} \mathrm{C}$ (Scheme 1). It is essential to use $N$-methyl-2pyrrolidone (NMP) as a solvent to obtain well-defined products. The copolymers P1-P5 were obtained as orange to brown, highly viscous liquids that were soluble in aqueous solution at room temperature. Detailed characterization of the resulting polymers confirmed the incorporation of both monomers and formation of the hyperbranched structure as discussed in the following. The ${ }^{1} \mathrm{H}$ NMR spectrum ( $c f$. ESI, Fig. S1 $\dagger$ ) shows the resonances of the polyether backbone $(\delta=$ 3.19-3.76 ppm), the hydroxyl groups $(\delta=4.40-4.80 \mathrm{ppm})$ and the characteristic signals of the ferrocene units at $\delta=4.14$ and $4.23 \mathrm{ppm}$. The comparison of the integrals of the initiator resonances $\left(\mathrm{CH}_{3}: \delta=0.80 \mathrm{ppm}\right.$ and $\left.\mathrm{CH}_{2}: \delta=1.20 \mathrm{ppm}\right)$ with the hydroxyl and ferrocene signals, respectively, allows to determine the incorporation ratio of both monomers and the molecular weight of the polymer. The calculated molecular weights are in the range of 3500 to $7300 \mathrm{~g} \mathrm{~mol}^{-1}$ with a fcGE content of 6.1 to $12.0 \%$ and are in agreement with the theoretical values based on the amount of TMP initiator employed. Higher comonomer contents are certainly feasible, however, have not been targeted, since water-soluble hyperbranched polymers were in the focus of this work. The molecular weights determined via SEC are consistently lower than the NMR values (1600 and $2100 \mathrm{~g} \mathrm{~mol}^{-1}, c f$. Fig. S4†). This can be attributed to the use of

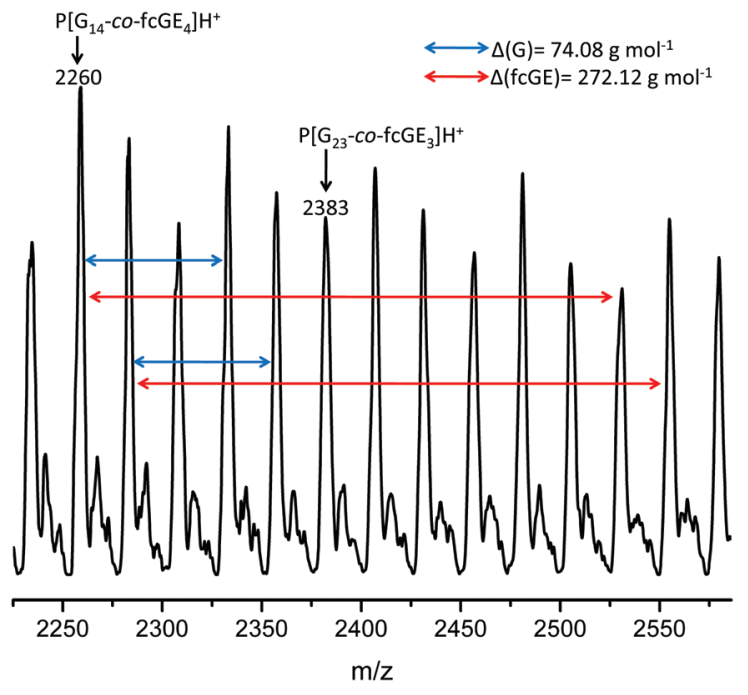

Fig. 1 MALDI ToF MS of hbP[G $\left.\mathrm{G}_{36}-\mathrm{co}-\mathrm{fcGE}_{5.0}\right](\mathrm{P} 2)$.

linear PEG standards. The hyperbranched topology, the multiple hydroxyl groups and the additional ferrocene units have an impact on the hydrodynamic volume of the polymers and therefore, a change in elution times and consequent underestimation of molecular weight can occur, which is often observed for hyperbranched polymers. ${ }^{35}$

As the controlled synthesis of hbPG is limited to $6000 \mathrm{~g}$ $\mathrm{mol}^{-1}{ }^{18}$ the synthesis of polymers with higher molecular weights is conducted by a two-step approach using a low molecular weight hbPG macroinitiator. ${ }^{23}$ The copolymer P5 was synthesized using P1: after deprotonation (10\% of hydroxyl groups) a mixture of glycidol and 5\% fcGE was slowly added via a syringe pump. The obtained copolymer showed a strong increase in molecular weight $\left(M_{\mathrm{n}}=12300 \mathrm{~g} \mathrm{~mol}^{-1}\right)$ under preservation of the low molecular weight dispersity $(D=1.69)$. Furthermore, the targeted fcGE content of 5\% was obtained according to NMR characterization.

Matrix-assisted laser desorption/ionization time-of-flight mass spectrometry (MALDI-ToF MS) was performed to confirm incorporation of both monomers in the polymer backbone. Fig. S5 $\uparrow$ shows the mass spectrum of sample P2, and Fig. 1 a zoom into the spectrum: the molecular masses of glycidol (74.08 $\mathrm{g} \mathrm{mol}^{-1}$, blue) and fcGE (272.12 $\mathrm{g} \mathrm{mol}^{-1}$, red) can be assigned, evidencing successful copolymerization of both monomers. Furthermore, two signals are marked for the corresponding copolymer cationized with $\mathrm{H}^{+}$.

\section{Degree of branching}

The degree of branching (DB) is an important parameter of hyperbranched polymers, which is 0 for linear polymers and 1 for maximum branching. Inverse gated (IG) ${ }^{13} \mathrm{C}$ NMR spectroscopy enables the integration of ${ }^{13} \mathrm{C}$ resonances and thus calculation of the relative abundance of the different structural units. Glycidol can be incorporated as dendritic (D), linear-1,3 


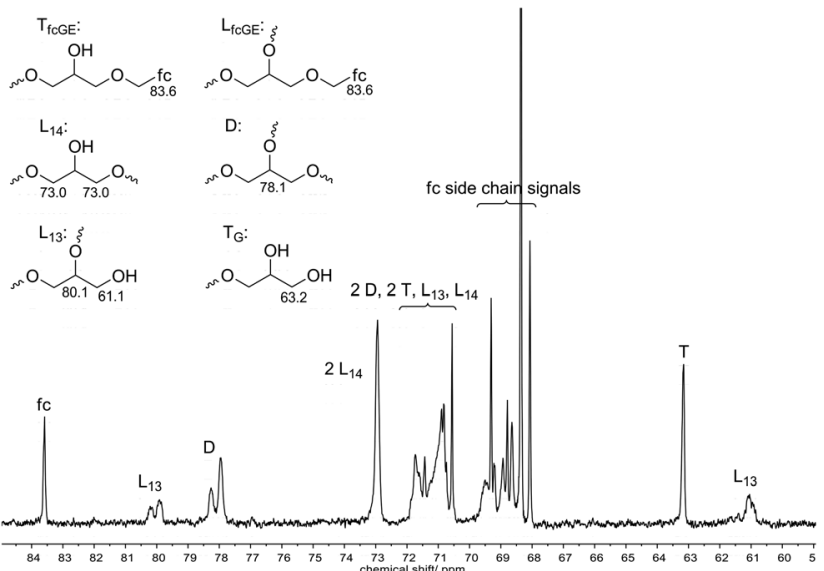

Fig. 2 Inverse-Gated (IG) ${ }^{13} \mathrm{C}$ NMR spectrum (100 MHz, DMSO- $d_{6}$ ) of $\mathrm{hbP}\left[\mathrm{G}_{36}-\mathrm{co}-\mathrm{fcGE}_{5.0}\right](\mathrm{P} 2)$.

$\left(\mathrm{L}_{1,3}\right)$, linear-1,4 $\left(\mathrm{L}_{1,4}\right)$ and terminal $(\mathrm{T})$ unit, whereas fcGE can only be incorporated as linear $\left(\mathrm{L}_{\mathrm{fCGE}}\right)$ or terminal $\left(\mathrm{T}_{\mathrm{fcGE}}\right)$ unit as depicted in Fig. 2. The DB was calculated using eqn (1), ${ }^{36,37}$ which is feasible for the copolymerization of glycidol with a cyclic $\mathrm{AB}$ monomer like fcGE.

$$
\mathrm{DB}=\frac{2 \mathrm{D}}{2 \mathrm{D}+\mathrm{L}_{13}+\mathrm{L}_{14}+\mathrm{L}_{\mathrm{fcGE}}}
$$

The calculated DB values are summarized in Table 1 and the relative integrals of the different structural units are shown in Table S1. $\dagger$ The DB decreases from 0.54 to 0.40 for increasing fcGE content, as expected for the copolymerization of an $\mathrm{AB}$ and $\mathrm{AB}_{2}$ monomer. ${ }^{24}$

The differentiation between $\mathrm{L}_{\mathrm{fCGE}}$ and $\mathrm{T}_{\mathrm{fcGE}}$ units is not possible in the NMR spectra. Therefore all incorporated fcGE units were considered as linear units for the calculation of the $\mathrm{DB}$, which is realistic, considering that fcGE monomer was only used as the minority fraction. However, to determine the error of this assumption the DB was also calculated based on the assumption that all fcGE units were incorporated at the termini. In this case, the DB values are 5 to $10 \%$ higher than the values given in Table 1, without influencing the observed trend.

\section{Thermal properties}

Differential scanning calorimetry (DSC) measurements were performed to obtain information on the thermal properties of the copolymers (Table 1). The glass transition temperature $\left(T_{\mathrm{g}}\right)$ for the hbPG homopolymer is detected in the range between -19 to $-26{ }^{\circ} \mathrm{C},{ }^{22}$ whereas the linear $\mathrm{P}(\mathrm{fcGE})$ homopolymer shows a $T_{\mathrm{g}}$ of $-8{ }^{\circ} \mathrm{C} .{ }^{6}$ For the copolymers P1 to P3 the $T_{\mathrm{g}}$ increases with increasing fcGE content and increasing molecular weight (from -24 to $-15{ }^{\circ} \mathrm{C}$, Table 1). It is remarkable that the fc groups hardly affect the $T_{\mathrm{g}}$ of the hyperbranched polyethers in comparison to hbPG. Ferrocene is a sterically demanding substituent and therefore can be expected to impede bond rotation, which explains these observations. However, P4 shows the lowest $T_{\mathrm{g}}$ of all polymers although this sample exhibits the highest fcGE content. We assume that in this case different effects like molecular weight, comonomer content and degree of branching lead to a non-linear trend of the $T_{\mathrm{g}} \mathrm{s}$.

\section{Redox and solubility properties in water}

The redox-responsive properties of the copolymers have been studied by cyclovoltammetry. Applying a cyclic potential to a polymer solution, the reversible redox-activity of fc can be studied. All synthesized copolymers were analyzed via cyclic voltammetry at three different scan rates $\left(50 \mathrm{mV} \mathrm{s}^{-1}, 100 \mathrm{mV}\right.$ $\mathrm{s}^{-1}$ and $150 \mathrm{mV} \mathrm{s}^{-1}$; Fig. 3 and S9-S11†). The copolymers were dissolved in water at a concentration of $5 \mathrm{~g} \mathrm{~L}^{-1}$ with $0.1 \mathrm{M}$ potassium chloride as a conducting salt. The reversible redoxactivity of $\mathrm{fc}$ is visualized by plotting the cyclic potential against the measured current. The graphs show the characteristic oxidation and reduction peaks of fc. Reversibility of the electrochemical reduction is indicated by the symmetry of the measured cycles, whereas the potentials at the current maxima $\left(E^{\mathrm{ox}}=0.2 \mathrm{~V}\right)$ and minima $\left(E^{\mathrm{red}}=0.1 \mathrm{~V}\right)$ are independent of the scan rates for all samples. Neither degradation nor consecutive reactions were detected in several consecutive measured cycles.

Table 1 Characterization data for hbP[G-co-fcGE] copolymers

\begin{tabular}{|c|c|c|c|c|c|c|c|c|}
\hline No. & Sample & $\begin{array}{l}M_{\mathrm{n}}{ }^{a} \\
{\left[\mathrm{~g} \mathrm{~mol}^{-1}\right]}\end{array}$ & $\begin{array}{l}M_{\mathrm{n}}^{b} \\
{\left[\mathrm{~g} \mathrm{~mol}^{-1}\right]}\end{array}$ & $\oplus^{b}$ & $\begin{array}{l}\text { fcGE } \\
{[\mathrm{mol} \%]}\end{array}$ & $\mathrm{DB}^{c}$ & $\begin{array}{l}T_{\mathrm{g}}{ }^{d} \\
{\left[{ }^{\circ} \mathrm{C}\right]}\end{array}$ & $\begin{array}{l}T_{\mathrm{c}}^{e} \\
{\left[{ }^{\circ} \mathrm{C}\right]}\end{array}$ \\
\hline P1 & $\mathrm{hbP}\left[\mathrm{G}_{37}-\mathrm{co}-\mathrm{fcGE}_{2.4}\right]$ & 3500 & 1600 & 1.56 & 6.1 & 0.54 & -22 & - \\
\hline P3 & $\mathrm{hbP}\left[\mathrm{G}_{67}-\mathrm{co}-\mathrm{fcGE}_{8.2}\right]$ & 7300 & 2000 & 1.55 & 10.9 & 0.49 & -15 & 49 \\
\hline $\mathrm{P} 4$ & $\mathrm{hbP}\left[\mathrm{G}_{46}-\mathrm{co}-\mathrm{fcGE}_{6.3}\right]$ & 5300 & 1700 & 1.40 & 12.0 & 0.40 & -24 & 45 \\
\hline P5 & $\mathrm{hbP}\left[\mathrm{G}_{140}-c o-\mathrm{fcGE}_{7}\right]$ & 12300 & 6800 & 1.69 & 4.8 & 0.62 & -24 & - \\
\hline
\end{tabular}

${ }^{a} M_{\mathrm{n}}$ determined from ${ }^{1} \mathrm{H}$ NMR spectroscopy in DMSO- $d_{6}$ by end-group analysis. ${ }^{b} M_{\mathrm{n}}$ determined via size exclusion chromatography in DMF $v s$. PEG standards using the RI-signal detection, $D=M_{\mathrm{w}} / M_{\mathrm{n}}$. ${ }^{c} \mathrm{DB}$ determined via IG ${ }^{13} \mathrm{C}$ NMR spectroscopy (DMSO- $d_{6} ; 100 \mathrm{MHz}$ ) according to eqn (1). ${ }^{d}$ Glass transition temperature $\left(T_{\mathrm{g}}\right)$ determined from differential scanning calorimetry (heating/cooling rate $10 \mathrm{~K} \mathrm{~min}{ }^{-1}$; second heating run). ${ }^{e}$ Cloud point temperature for a $5 \mathrm{mg} \mathrm{mL}^{-1}$ solution of the copolymer in deionized water, measured with a heating rate of $5 \mathrm{~K} \mathrm{~min}^{-1}$. 


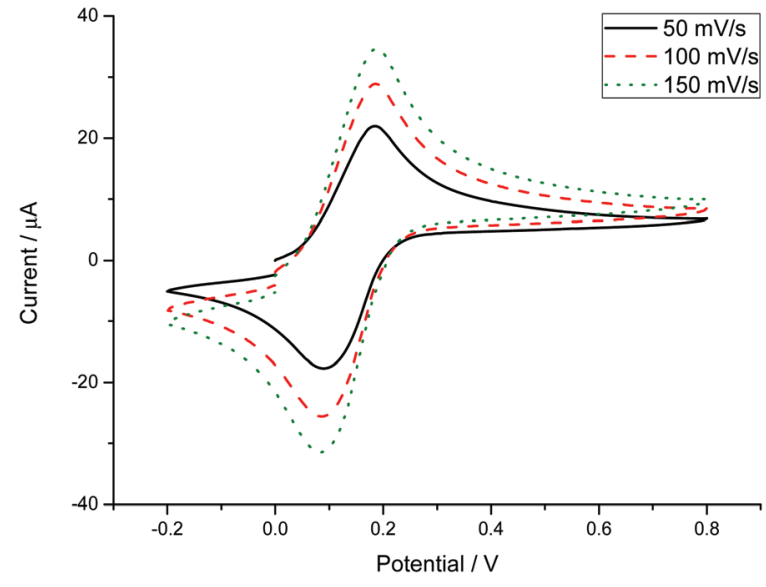

Fig. 3 Cyclic voltammograms of $h b P\left[G_{67}-\mathrm{CO}-\mathrm{fcGE}_{8.2}\right]$ (P3) at different scan rates $\left(\mathrm{H}_{2} \mathrm{O}, 5 \mathrm{~g} \mathrm{~L}^{-1} \mathrm{P} 1,0.1 \mathrm{M} \mathrm{KCl}\right)$.

It has been reported that copolymers of EO and a hydrophobic comonomer exhibit lower hydrophilicity compared to PEG. Materials of this type show cloud point temperatures below $100{ }^{\circ} \mathrm{C}$ or may be insoluble in aqueous solution. In the case of fcGE, the cloud point temperatures $\left(T_{\mathrm{c}}\right)$ of the linear $\mathrm{P}$ [fcGE-co-EO] copolymers were varied from 7.2 to $82.2{ }^{\circ} \mathrm{C} .{ }^{5}$ Copolymers of glycidol and a hydrophobic comonomer show similar properties, however, due to the large number of hydroxyl groups the overall hydrophilicity is higher. The cloud point temperatures of the herein synthesized $\mathrm{hbP}[\mathrm{G}-\mathrm{co}-\mathrm{fcGE}]$ copolymers can be varied from 45 to $60^{\circ} \mathrm{C}$. However, to obtain the same $T_{\mathrm{c}}$ in case of the hb polymers a higher amount of fcGE could be incorporated compared to the linear PEG-copolymers. Sample P1 and P5 with the lowest amounts of fcGE are water-soluble over the whole temperature range. The turbidity measurements of P2, P3 and P4 are shown in Fig. 4.

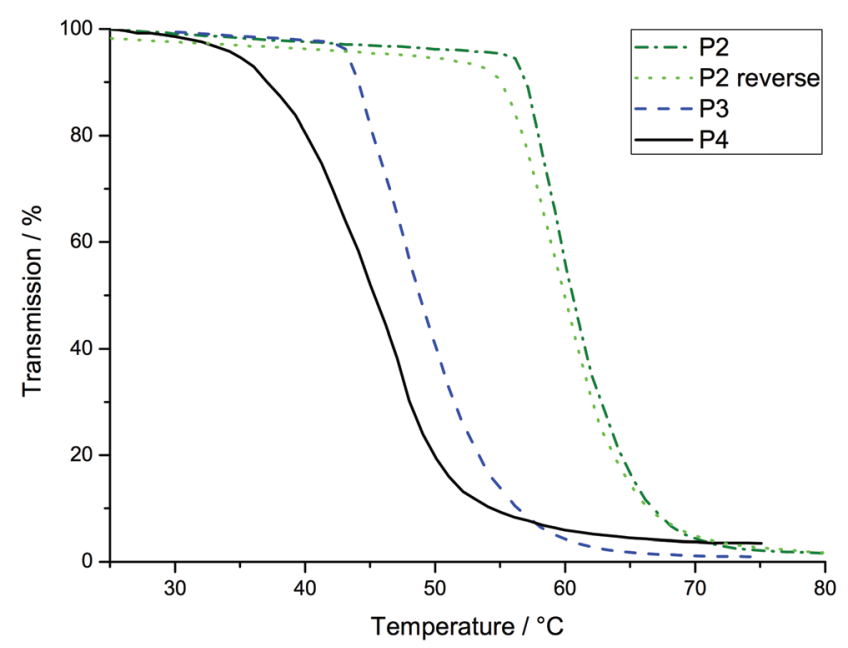

Fig. 4 Turbidimetry measurements of P2, P3 and P4 with a cooling curve for $\mathrm{P} 2$ in water $\left(5 \mathrm{~g} \mathrm{~L}^{-1}\right.$; heating/cooling rate $\left.5^{\circ} \mathrm{C} \mathrm{min}^{-1}\right)$.
A cooling curve of $\mathrm{P} 2$ is also shown to demonstrate the typical low hysteresis of the copolymers. The transitions for the hb copolymers are not as sharp as for the linear PEGcopolymers as reported previously, certainly due to the somewhat higher dispersity of the hb copolymers. Especially for low molecular weights and low amounts of fc incorporated, the absolute amount of fc among the individual polymer chains varies significantly, and consequently the solubility differs strongly.

Since fc is redox-active, the hydrophilicity of the copolymer can be increased by oxidation of $\mathrm{Fe}(\mathrm{II})$ to $\mathrm{Fe}(\mathrm{III})$, and the sandwich complex becomes positively charged. Thus, the $T_{\mathrm{c}}$ of a given copolymer can be shifted to a higher temperature by partial oxidation. The $T_{\mathrm{c}}$ of P3 is compared to the partly oxidized and fully oxidized P3 (Fig. 5). The partly oxidized sample shows a higher $T_{\mathrm{c}}$ (as expected), and in addition the transmittance does not reach $0 \%$, but remains at $c a$. $40 \%$, which indicates the presence of fully water-soluble copolymers in the mixture. The oxidized P3 is water-soluble, and the transmittance remains very high throughout the whole temperature range.

Mößbauer spectroscopy has hardly been employed to characterize the degree of oxidation of redox-active dendritic polymers. ${ }^{4}$ The influence of oxidation on the aqueous solubility of the hyperbranched copolymers was further quantified by Mößbauer spectroscopy in aqueous solution. The copolymer P3 exhibits an original cloud point of $49{ }^{\circ} \mathrm{C}$, after partial oxidation with silver triflate the $T_{\mathrm{c}}$ increased to $66{ }^{\circ} \mathrm{C}$. After full oxidation of the copolymer P3 no cloud point was detectable. Quantitative analysis of the three samples (not oxidized P3, partially oxidized P3 and fully oxidized P3) via Mößbauer spectroscopy provided the ratio of ferrocenium ions $\left(\mathrm{Fe}^{3+}\right)$ to ferrocene $\left(\mathrm{Fe}^{2+}\right)$, since ferrocene shows a doublet with a quadrupole splitting at $2.37 \mathrm{~mm} \mathrm{~s}^{-1}$ and ferrocenium salts show a

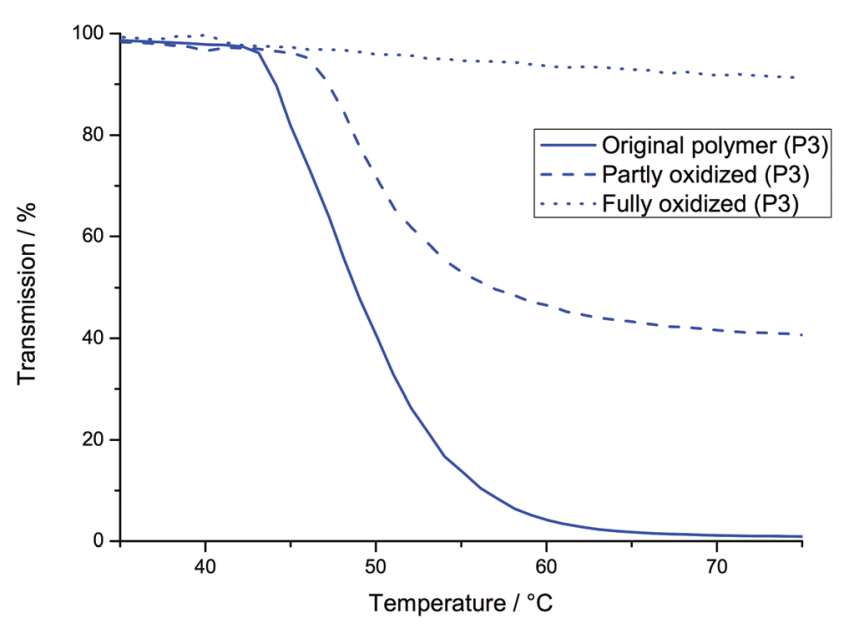

Fig. 5 Turbidity measurements of P3 upon oxidation. After partly (53\% of fc units) oxidation of the starting polymer (P3, line) the cloud point temperature is lowered $(\mathrm{P} 3$, dashed) until finally the thermoresponsive behavior completely disappears (dotted) for $100 \%$ oxidation $\left(5 \mathrm{~g} \mathrm{~L}^{-1}\right.$; heating/cooling rate $5^{\circ} \mathrm{C} \mathrm{min}^{-1}$ ). 


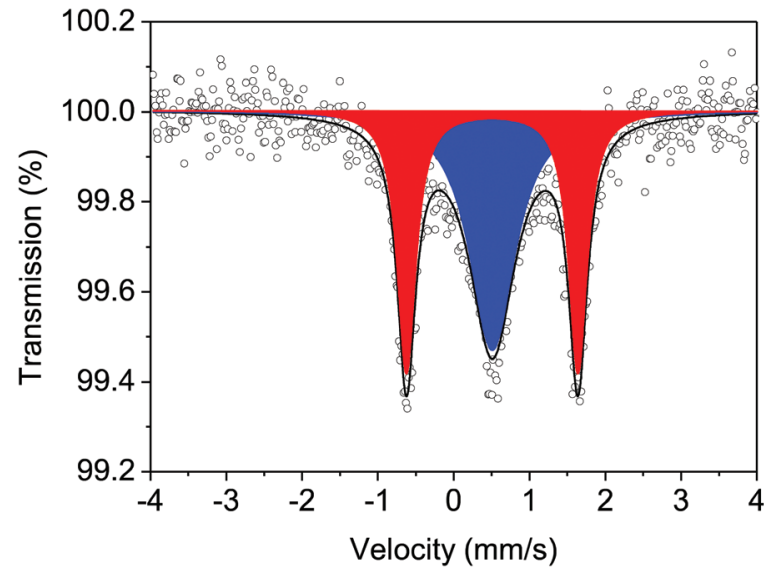

Fig. 6 Mößbauer spectrum of the partially $(53 \pm 4 \%)$ oxidized copolymer P3 showing Fe(II) doublet (red) and Fe(III) singlet (blue).

singlet. ${ }^{38}$ The amount of ferrocenium ions was determined to be $0 \%$ for the non-oxidized sample P3 (Fig. S6 $\dagger$ ), $53 \pm 4 \%$ for the partially oxidized (Fig. 6) and $100 \%$ for the fully oxidized (Fig. S7†) sample. The determined $T_{\mathrm{c}} \mathrm{S}$ were plotted against the amounts of ferrocenium ions to visualize the dependency (Fig. S8\%). A similar dependency of the $T_{\mathrm{c}}$ and the concentration of ferrocenium ions for other copolymers is likely.

\section{Conclusions}

In summary, we have introduced the first water-soluble hyperbranched polyether copolymers with redox-active ferrocene units. Capitalizing on the one-pot $\mathrm{AB}+\mathrm{AB}_{2}$ copolymerization under slow monomer addition conditions, the copolymerization of glycidol and fcGE via anionic ROMBP provided copolymers with molecular weights of 3500 to $12300 \mathrm{~g} \mathrm{~mol}^{-1}$ with low molecular weight dispersities $(\theta=1.40$ to 1.69 ). Detailed characterization of the polymers confirms successful copolymerization of both monomers, resulting in water-soluble hyperbranched ferrocene-containing polymers. Additionally, the polymers exhibit multi-stimuli responsive behavior. The cloud point temperature could be adjusted by variation of the fcGE comonomer content, as well as the amount of oxidized iron-centers. These materials significantly enhance the scope of ferrocene-based polymers, as they exhibit a large number of hydroxyl groups, enabling further functionalization and an overall higher degree of hydrophilicity. The latter resulted in increased cloud point temperatures of the fc-containing PGs compared to the linear PEG analogues. Thus higher amounts of fcGE can be incorporated with lower influence on the solubility of the polymers. Mößbauer spectroscopy of the aqueous polymer solutions was used to correlate the degree of oxidation of fc with an increase of the cloud point temperatures. These findings render the novel polymers promising for a variety of purposes, e.g., as polymer therapeutics, or in detection or sensing applications.

\section{Acknowledgements}

The authors thank Prof. Dr Katharina Landfester and Dr Vadim Ksenofontov for support. R. K. thanks the Graduate School of Excellence Materials Science in Mainz "MAINZ" for financial support. The authors thank Fabienne Ludwig and Luka Decker for technical assistance and Dr Elena BergerNicoletti for MALDI-ToF MS measurements. F. R. W. thanks the Max Planck Graduate Center for support.

\section{References}

1 D. A. Rider and I. Manners, Polym. Rev., 2007, 47, 165195.

2 Y. Ni, R. Rulkens and I. Manners, J. Am. Chem. Soc., 1996, 118, 4102-4114.

3 F. S. Arimoto and A. C. Haven, J. Am. Chem. Soc., 1955, 77, 6295-6297.

4 J. C. Lai, D. P. Vanderpool, M. Good, R. Prado and C. U. Pittman, Macromolecules, 1970, 3, 746-754.

5 C. Tonhauser, A. Alkan, M. Schömer, C. Dingels, S. Ritz, V. Mailänder, H. Frey and F. R. Wurm, Macromolecules, 2013, 46, 647-655.

6 A. Alkan, A. Natalello, M. Wagner, H. Frey and F. R. Wurm, Macromolecules, 2014, 47, 2242-2249.

7 A. Alkan, L. Thomi, T. Gleede and F. R. Wurm, Polym. Chem., 2015, 6, 3617-3624.

8 W. Wu, R. Tang, Q. Li and Z. Li, Chem. Soc. Rev., 2015, 44, 3997-4022; F. Wurm, F. J. L. Villanueva and H. Frey, J. Polym. Sci. Part A; Polym. Chem., 2009, 47, 25182529.

9 S. Lhenry, J. Jalkh, Y. R. Leroux, J. Ruiz, R. Ciganda, D. Astruc and P. Hapiot, J. Am. Chem. Soc., 2014, 136, 17950-17953.

10 F. Wurm, S. Hilf and H. Frey, Chem. - Eur. J., 2009, 15, 9068-9077.

11 J. Kong, T. Schmalz, G. Motz and A. H. E. Müller, Macromolecules, 2011, 44, 1280-1291.

12 Q. Tan, L. Wang, L. Ma, H. Yu, Q. Liu and A. Xiao, Macromolecules, 2009, 42, 4500-4510.

13 J. Shi, C. J. W. Jim, F. Mahtab, J. Liu, J. W. Y. Lam, H. H. Y. Sung, I. D. Williams, Y. Dong and B. Z. Tang, Macromolecules, 2010, 43, 680-690.

14 Y. H. Kim and O. W. Webster, J. Am. Chem. Soc., 1990, 112, 4593-4594.

15 C. Gao and D. Yan, Prog. Polym. Sci., 2004, 29, 183-275.

16 B. I. Voit and A. Lederer, Chem. Rev., 2009, 109, 59245973.

17 F. Vögtle, G. Richardt and N. Werner, Dendrimer Chemistry, WILEY-VCH, Weinheim, 2009.

18 D. Wilms, S.-E. Stiriba and H. Frey, Acc. Chem. Res., 2010, 43, 129-141.

19 R. Klein and F. R. Wurm, Macromol. Rapid Commun., 2015, 36, 1147-1165.

20 R. K. Kainthan, J. Janzen, E. Levin, D. V. Devine and D. E. Brooks, Biomacromolecules, 2006, 7, 703-709. 
21 M. Calderón, M. A. Quadir, S. K. Sharma and R. Haag, Adv. Mater., 2010, 22, 190-218.

22 A. Sunder, R. Hanselmann, H. Frey and R. Mülhaupt, Macromolecules, 1999, 32, 4240-4246.

23 D. Wilms, F. Wurm, J. Nieberle, P. Böhm, U. Kemmer-Jonas and H. Frey, Macromolecules, 2009, 42, 3230-3236.

24 A. Sunder, H. Türk, R. Haag and H. Frey, Macromolecules, 2000, 33, 7682-7692.

25 M. Schömer, J. Seiwert and H. Frey, ACS Macro Lett., 2012, 1, 888-891.

26 C. Kojima, K. Yoshimura, A. Harada, Y. Sakanishi and K. Kono, Bioconjugate Chem., 2009, 20, 1054-1057.

27 C. Kojima, K. Yoshimura, A. Harada, Y. Sakanishi and K. Kono, Polym. Sci., Part A: Polym. Chem., 2010, 48, 40474054 .

28 X. Sun, Y. Zhou and D. Yan, Macromol. Chem. Phys., 2010, 211, 1940-1946.

29 C. Tonhauser, C. Schüll, C. Dingels and H. Frey, ACS Macro Lett., 2012, 1, 1094-1097.
30 R. A. Shenoi, J. K. Narayanannair, J. L. Hamilton, B. F. L. Lai, S. Horte, R. K. Kainthan, J. P. Varghese, K. G. Rajeev, M. Manoharan and J. N. Kizhakkedathu, J. Am. Chem. Soc., 2012, 134, 14945-14957.

31 R. A. Shenoi, B. F. L. Lai, M. I. ul-haq, D. E. Brooks and J. N. Kizhakkedathu, Biomaterials, 2013, 34, 6068-6081.

32 S. Son, E. Shin and B.-S. Kim, Macromolecules, 2015, 48, 600-609.

33 K. Lagarec and D. G. Rancourt, Nucl. Instrum. Methods Phys. Res., Sect. B, 1997, 129, 266-280.

34 C. Schüll, T. Gieshoff and H. Frey, Polym. Chem., 2013, 4, 4730-4736.

35 A. Lederer, W. Burchard, A. Khalyavina, P. Lindner and R. Schweins, Angew. Chem., Int. Ed., 2013, 52, 4659-4663.

36 D. Hölter, A. Burgath and H. Frey, Acta Polym., 1997, 48, 30-35.

37 H. Frey and D. Hölter, Acta Polym., 1999, 50, 67-76.

38 G. K. Wertheim and R. H. Herber, J. Chem. Phys., 1963, 38, 2106-2111. 